\title{
Promoting Awareness on Cerebrovascular Disease in Young Adults is a Work in Progress
}

\author{
Aleksandra Pikula* \\ Department of Neurology, Boston University School of Medicine, Boston, MA 02118, USA
}

Over the past decades, the overall stroke incidence has been declining, but among young adults the stroke incidence is increasing. Population and hospital-based studies have raised concerns about the increasing prevalence of traditional stroke risk factors among young adults and its consequence on stroke incidence, stroke recurrence and stroke related morbidity and mortality $[1,2]$.

Stroke events in individuals younger than 50 years of age are considered "strokes in young adults". Based on the US Nationwide Inpatient Sample data from 1993-2008, hospital admissions among patients 14-44 years old with diagnosis of ischemic stroke have increased by $\sim 50 \%$. The same data show concurrent increase in prevalence of traditional stroke risk factors among young for obesity, dyslipidemia, hypertension and smoking [3,4]. Due to popular belief that stroke is a disease of elderly, stroke symptoms among young are commonly misdiagnosed in the acute settings. The young stroke patients are also less likely to use pre-hospital stroke pathway systems [5]. However, the severity of ischemic stroke is milder [6], and the prognosis after stroke in young adults is more favorable then in the elderly [7]. Nevertheless, the long-term psychosocial consequences are greater and the risk for recurrence due to longer lifetime expectancy is much higher [1,8-10].

With introduction of sensitive brain imaging techniques it had been established that the burden of cerebrovascular disease is far greater than that of overt clinical disease [11]. In large population-based studies, the MRI findings of subclinical vascular brain injury such as presence of covert brain infarcts (CBIs), white matter hyperintensities (WMHs) and cerebral microbleeds (CMBs) are common. The prevalence of CBIs and WMHs ranges from $10-20 \%$ in subjects $<65$ years of age, and frequency of CMBs while limited by number of studies available was $6.5-8 \%$ in $<50$ years old otherwise healthy subjects $[12,11]$. While these subclinical findings do not cause abrupt clinical symptoms, they are often linked to cognitive decline and motor function impairment, and have been associated with an increased risk of subsequent stroke and dementia [11]. The complexity of MRI techniques and inconsistency in the definitions of these MRI measurements limits systematic examination of their frequencies and its relevance on the functional and cognitive outcomes in young adults with ischemic stroke. As a result, there are only a few studies that evaluated this in some detail.

Utilizing quantitative scores that are based on presence or absence of SBIs and on the degree of WMHs (mild, moderate or severe) observed on brain MRI, the Helsinki Stroke Registry retrospectively examined 669 predominantly white patients with first -ever ischemic stroke. SBIs were present in $13 \%$ and WMHs in $7 \%$ of all patients. Most patients had mild to moderate degree of WMHs. The risk factors for higher prevalence of SBIs and WMHs were older age, type $1 \mathrm{DM}$ and obesity, but not hypertension as usually observed in middle aged or elderly population. In multivariate analysis, the presence of SBIs independently increased the risk of recurrent stroke, and moderate to severe WMHs were independently associated with a greater risk of death $(\mathrm{OR}=3.43)$ [13].

From the single-center French retrospective study, among 170 adults (18-50 years old) with first-ever ischemic stroke, $28 \%$ patients had silent ischemic lesions (SILs). Over the mean follow-up of $\sim 25$ months, $23 \%$ of patients with SILs had a recurrent stroke vs. $6.5 \%$ without SILs, $\mathrm{p}=0.003$. After adjusting for vascular risk factors, recurrent stroke was associated with presence of SILs (HR 3.2, $\mathrm{p}=0.002$ ), and more so with the presence of combination of SBIs and WMHs (HR 7.3, p=0.003) [14].

Data published in this month's issue of Neurology arrives from the largest prospective study of stroke in young adults with Fabry disease (SIFAP1 study) that enrolled young adults with first ever TIA or stroke aged 18-55 years (over 2000 subjects with ischemic stroke and TIA). They observed equally high prevalence of WMH (46\% of all) with the severity positively associated with older age ( $>45$ years). The old infarcts were present in $8 \%$ of patients younger than 35 years, while in the age groups 35-44 and older than 44, the prevalence of old infarcts was $12 \%$ and $25 \%$, respectively.

Intriguingly, this rate was double the one of SBIs prevalence among young adults with first-ever ischemic stroke observed in Helsinki study. The prevalence of traditional risk factors was high and the prevalence of Fabry disease in the same study as reported earlier was 0.5\% [15].

Interestingly, Fabry disease frequently presents with clinical symptoms and MRI lesions that are often misdiagnosed as multiple sclerosis [16]. In this study, the prevalence of WMH among patients diagnosed with Fabry disease was unclear. There was also no discussion on how this could influence the overall study findings. It is worth underlining that SBIs and WMHs are frequently seen in patients with other more common conditions such as migraine or multiple sclerosis, and/or with less common but other determined stroke etiologies such as CADASIL (cerebral autosomal dominant arteriopathy with subcortical infarcts and leukoencephalopathy), MELAS (mitochondrial encephalomyopathy, lactic acidosis, and stroke-like episodes), lupus, various encephalopathies and vasculitidies. So, when interpreting data from this and all of the mentioned studies, it is important to notice that patients with these conditions should have been accounted for or excluded from the analysis.

If we recognize these shortcomings, along with the differences in the study populations and designs, these reports still provide an intriguing observation of strikingly high prevalence of so-called silent

*Corresponding author: Aleksandra Pikula, Assistant Professor, Department of Neurology, Boston University School of Medicine, 715 Albany Street, D-312, Boston, MA 02118-2526, USA, Tel: (617)-638-5435; Fax: (617) 638-8465; E-mail: alpikula@bu.edu

Received November 28, 2013; Accepted November 30, 2013; Published December 07, 2013

Citation: Pikula A (2013) Promoting Awareness on Cerebrovascular Disease in Young Adults is a Work in Progress. J Neurol Neurophysiol 5: e115. doi:10.4172/2155-9562.1000e115

Copyright: (c) 2013 Pikula A. This is an open-access article distributed under the terms of the Creative Commons Attribution License, which permits unrestricted use, distribution, and reproduction in any medium, provided the original author and source are credited. 
Citation: Pikula A (2013) Promoting Awareness on Cerebrovascular Disease in Young Adults is a Work in Progress. J Neurol Neurophysiol 5: e115. doi:10.4172/2155-9562.1000e115

brain infarcts and WMHs among young adults with ischemic stroke. Given that presence of WMHs on MRI are associated with an increased risk of stroke in the general population and in high risk populations with a history of stroke [11], once documented during diagnostic investigations it should prompt extensive exploration of possible etiologies as well as aggressive treatment of concurrent risk factors.

Consequently, it is not only the increased incidence of stroke and stroke risk factors that we need to take into account, but also the high prevalence of covert cerebrovascular disease in young adults. Further research should focus on all aspects of overt and covert cerebrovascular disease, and on rising public awareness and providing better access to primary and secondary stroke preventive care for young adults.

\section{References}

1. Putaala J, Curtze S, Hiltunen S, Tolppanen H, Kaste M, et al. (2009) Causes of death and predictors of 5-year mortality in young adults after first-ever ischemic stroke: the Helsinki Young Stroke Registry. Stroke 40: 2698-2703.

2. Putaala J, Metso AJ, Metso TM, Konkola N, Kraemer Y, et al. (2009) Analysis of 1008 consecutive patients aged 15 to 49 with first-ever ischemic stroke: The helsinki young stroke registry. Stroke; a journal of cerebral circulation. 40:11951203.

3. George MG, Tong X, Kuklina EV, Labarthe DR (2011) Trends in stroke hospitalizations and associated risk factors among children and young adults, 1995-2008. Ann Neurol 70: 713-721.

4. Kissela BM, Khoury JC, Alwell K, Moomaw CJ, Woo D, et al. (2012) Age at stroke: temporal trends in stroke incidence in a large, biracial population. Neurology 79: 1781-1787.

5. Kuruvilla A, Bhattacharya P, Rajamani K, Chaturvedi S (2011) Factors associated with misdiagnosis of acute stroke in young adults. Journal of stroke and cerebrovascular diseases: the official journal of National Stroke Association 20: 523-527.
6. Toni D, Ahmed N, Anzini A, Lorenzano S, Brozman M, et al. (2012) Intravenous thrombolysis in young stroke patients: results from the SITS-ISTR. Neurology 78: 880-887.

7. Varona JF, Bermejo F, Guerra JM, Molina JA (2004) Long-term prognosis of ischemic stroke in young adults. Study of 272 cases. J Neurol 251: 1507-1514.

8. Rutten-Jacobs LC, Maaijwee NA, Arntz RM, Schoonderwaldt HC, Dorresteijn LD, et al. (2013) Long-term risk of recurrent vascular events after young stroke: The FUTURE study. Ann Neurol 74: 592-601.

9. Schaapsmeerders P, Maaijwee NA, van Dijk EJ, Rutten-Jacobs LC, Arntz RM et al. (2013) Long-term cognitive impairment after first-ever ischemic stroke in young adults. Stroke 44: 1621-1628.

10. Rutten-Jacobs LC, Arntz RM, Maaijwee NA, Schoonderwaldt HC, Dorresteijn LD, et al. (2013) Long-term mortality after stroke among adults aged 18 to 50 years. JAMA 309: 1136-1144

11. Debette S, Markus HS (2010). The clinical importance of white matte hyperintensities on brain magnetic resonance imaging: Systematic review and meta-analysis. Bmj 341: c3666.

12. Shoamanesh A, Kwok CS, Benavente O (2011) Cerebral microbleeds: histopathological correlation of neuroimaging. Cerebrovasc Dis 32: 528-534.

13. Putaala J, Kurkinen M, Tarvos V, Salonen O, Kaste M, et al. (2009) Silent brain infarcts and leukoaraiosis in young adults with first-ever ischemic stroke. Neurology 72: 1823-1829.

14. Gioia LC, Tollard É, Dubuc V, Lanthier S, Deschaintre Y, et al. (2012) Silent ischemic lesions in young adults with first stroke are associated with recurrent stroke. Neurology 79: 1208-1214

15. Rolfs A, Fazekas F, Grittner U, Dichgans M, Martus P, et al. (2013) Acute cerebrovascular disease in the young: the Stroke in Young Fabry Patients study. Stroke 44: 340-349.

16. Böttcher T, Rolfs A, Tanislav C, Bitsch A, Köhler W, et al. (2013) Fabry disease - underestimated in the differential diagnosis of multiple sclerosis? PLoS One 8: e71894. 